Personality and Mental Health

2: 171-182 (2008)

Published online in Wiley InterScience

(www.interscience.wiley.com) DOI: 10.1002/pmh.43

\title{
Tattoos and antisocial personality disorder
}

WILLIAM CARDASIS ${ }^{1}$, ALISSA HUTH-BOCKS ${ }^{2}$ AND KENNETH R. SILK ${ }^{3},{ }^{1}$ Center for Forensic Psychiatry, Michigan Department of Community Health, USA; ${ }^{2}$ Department of Psychology, Eastern Michigan University, USA; ${ }^{3}$ Department of Psychiatry, University of Michigan, USA

\section{ABSTRACT}

Objective The relationship of tattoos to the diagnosis of antisocial personality disorder (ASPD) was explored in a forensic psychiatric inpatient hospital setting. It was hypothesized that a greater proportion of forensic inpatients that possessed tattoos had ASPD than patients who did not possess tattoos.

Method Forensic male psychiatric inpatients $(\mathrm{N}=36)$ were administered a semi-structured interview to determine the presence of a tattoo. ASPD was determined by criteria on a Diagnostic and Statistical Manual of Mental Disorders-IV ASPD checklist. Demographic and background characteristics of the patients were collected, and details about each tattoo were obtained including a calculation of the surface area of each tattoo.

Results Significantly more forensic psychiatric inpatients with tattoos had a diagnosis of ASPD compared to patients without tattoos. Patients with ASPD also had a significantly greater number of tattoos, a trend toward having a greater percentage of their total body surface area tattooed, and were more likely to have a history of substance abuse than patients without ASPD. Tattooed subjects, with or without ASPD, were significantly more likely to have histories of substance abuse, sexual abuse and suicide attempts than non-tattooed patients.

Conclusions Forensic psychiatric inpatients with tattoos should be assessed carefully for the presence of ASPD as well as for substance abuse, sexual abuse and suicide attempts, factors having potentially significant influence on the assessment and treatment of such patients. Copyright (c) 2008 John Wiley $\mathcal{E}$ Sons, Ltd.

\section{Introduction}

Antisocial personality disorder (ASPD) is commonly diagnosed in patients in forensic psychiatric settings. Internal data from our facility, the Michigan Center for Forensic Psychiatry (CFP), reveal that over $25 \%$ of inpatients carry the diag- nosis. The presence of comorbid ASPD in patients with a primary diagnosis of a severe Axis I condition such as schizophrenia and bipolar disorder frequently complicates the clinical course (Hill, Neumann, \& Rogers, 2004; Kokkevi, Stefanis, Anastasopoulou, \& Kostogianni, 1998; Reich, 1997); Ross, Dermatis, Levounis, \& Galanter, 
2003). Challenges faced by clinicians treating forensic patients with comorbid ASPD include dealing with impulsive and manipulative behaviour that often may confound specific aspects of assessment, treatment and disposition. Further understanding of and assistance in identifying the antisocial condition is important, especially in forensic settings, in helping to provide more efficacious assessment and treatment planning.

\section{Conceptualization of the antisocial personality}

The antisocial personality concept initially emanated from psychodynamic theory. Within the psychodynamic perspective, the antisocial personality (also sometimes referred to historically as sociopathy or psychopathy) has been conceptualized as describing a range of psychological and behavioural phenomena (Hare \& Neumann, 2005; Hare, 2006), the primary feature relating to the individual's need to seek immediate gratification with little regard for other aspects of psychic functioning or for the demands of external reality. A concomitant feature is the individual's lack of empathy, particularly his disregard of the effect of his actions on others (Hare, Hart, \& Harpur, 1991). Because patients with ASPD are unable to control or modulate their impulses effectively, problematic and often unlawful behaviours may result. Patients with ASPD demonstrate a low tolerance for anxiety, lack of feelings of remorse and shallowness. They seek out external stimulation to avoid feelings of anxiety and other uncomfortable emotional states. They prefer action to words or thought. Pathological lying, cheating, stealing, physical aggression and drug abuse are not uncommon (Cleckley, 1950; MacKinnon \& Michels, 1971).

The concept of ASPD changed and narrowed with the publication of the Diagnostic and Statistical Manual of Mental Disorders-III (APA, 1980) and continued through the present DSM iteration, DSM-IV (APA, 1994; Hare et al., 1991). In the more recent DSM conceptualizations of ASPD, the notion of psychopathy has been lost. The more current DSM versions require that in order to meet the ASPD diagnosis, the individual must have met three of the subset of criteria that resemble Conduct Disorder in DSM-IV, and that this behaviour develops prior to age 15. Thus, the idea of the manipulative, scheming, dishonest politician or company executive would not meet the criteria if he or she failed to reveal a specific pattern of conduct disturbance prior to age 15 .

This paper will explore the presence and some of the characteristics of tattoos on male patients in a forensic psychiatric hospital and the relationship of those tattoos to the diagnosis of ASPD as measured by an ASPD checklist based on DSM-IV ASPD criteria. We will not try to determine whether the individual would be classified as a psychopath or a sociopath because trying to do that would confound the diagnostic process and add unwanted variability into the study.

From a dynamic perspective, patients with ASPD are thought to defend against painful emotions by utilizing defences such as acting out, denial of anxiety, isolation, displacement, projection and rationalization to diminish the guilt and social discomfort that they may experience. Such ego defences serve not only to protect the patient with ASPD from anxiety but also to shift the anxiety to others. Psychodynamic theory would suggest that in adult life, failure to develop mature ego functions is associated with inadequate or pathological attachments early in life and results in disturbed ego identity and object relations in adulthood (Kernberg, 1976). Patients with ASPD are thought to utilize outward physical body markings, such as tattoos, as a method of augmenting primitive psychological defence mechanisms (Popplestone, 1963).

The development of a clear ego identity requires a highly developed sense of body boundaries (Fisher, 1963). External threats to one's psychological integrity are defended against by several psychological defence mechanisms, some of which were listed previously. In one study exploring the psychodynamic implications of tattoos, Grumet (1983) indicated that the tattoo could be viewed as an artificial embellishment of the external body 
boundary, and in that context, could be considered an external attempt to strengthen one's sense of ego definition.

Prevalence and meaning of tattoos in different populations

In recent years, there has been an increase in persons with tattoos, with the images becoming increasingly eclectic. In a recent self-report survey of 247 men and 253 women, $24 \%$ of respondents had tattoos, and the presence of tattoos was equally common among both sexes. The presence of a tattoo was associated with a lack of religious affiliation, extended jail time, previous drinking, recreational drug use, and lower socioeconomic and educational status (Laumann \& Derrick, 2006). As a reference point, the prevalence of tattoos in the United States was reported to be about 16\% at the turn of the millennium (Anderson, 2006). The explanation for the increase in the overall prevalence of tattoos in the general population in recent years is unclear. Laumann and Derrick (2006) speculated that although tattooing is prohibited in a number of religious texts (e.g. the Old Testament and the Koran), it appears that less religious individuals in contemporary American society have more body art (tattoos or piercing) because of a lack of feeling of community and disconnection from religious institutions. Additional reasons for obtaining tattoos among the general population described by Wohlrab, Stahl and Kappeler (2007) have included the following categories: beauty, art and fashion (body art); individuality (create and maintain self-identity); personal narrative (expression of values and experiences); physical endurance (testing one's threshold for pain); group affiliations and commitment (membership or the wish to belong); resistance (against authority or provocation); spirituality and cultural tradition (emphasize cultural beliefs); addiction (related to release of endorphins and positive feeling); sexual motivation (expressing sexual affectations or sexuality); and no specific reason (impulsive, under the influence of psychoactive substances).
Although the prevalence of tattoos is unknown among general psychiatric patients, several studies have found an increased occurrence of a personality disorder among tattooed male psychiatric patients (Buhrich \& Morris, 1982; Gittleson, Wallen, \& Dawson-Butterworth, 1969). These studies looked at personality disorders in general, and not specifically at ASPD. Measey (1972) studied the psychiatric and social relevance of tattoos in Royal Navy detainees and found that the tattooed group showed a higher likelihood of personality disorder, in general, with the likelihood of a personality disorder also increasing as number of tattoos increased. No specific personality disorder type was defined in this study.

Tattooing is thought to be highly prevalent in prison populations (Manuel \& Retzlaff, 2002). Demello (1993) estimated that over half of all male prisoners in the United States have tattoos. Because tattooing in the prison setting is illegal to avoid the spread of blood borne disease, tattoos are usually monochromatic (black), made with improvised devices, needles, homemade ink, and are often visible on the face, neck and hands. In a sample of adolescent detainees, $29 \%$ had at least one tattoo, $15 \%$ had two or more tattoos and $21 \%$ had tattoos that were administered nonprofessionally (Braithwaite, Robillard, Woodring, Stephens, \& Arriola, 2001). Tattoos among prison populations may help serve the purpose of creating individual and group identities and asserting cultural differences among inmates (Demello, 1993). A recent study examining the association between tattoos and personality disorder in a prison sample found that the personality types more likely related to the presence of tattoos were antisocial, sadistic, negativistic and borderline personality pathology (Manuel \& Retzlaff, 2002).

\section{The present study}

While the previous studies looked at individuals in a prison setting, this study takes place in a forensic psychiatric hospital, to our knowledge the first study of tattoos in such a setting. The 
prevalence of ASPD in our forensic psychiatric facility is comparable to rates (29\%) reported in at least one study of prison inmates using the MCMIIII (Millon ${ }^{\mathrm{TM}}$ Clinical Multiaxial Inventory) antisocial personality scale (Manuel \& Retzlaff, 2002). Although comparing the prevalence of tattoos and ASPD in a forensic psychiatric hospital setting to rates in other populations, and in particular to a prison setting, may be useful, it is important to differentiate the forensic psychiatric population from persons in prison or correctional settings. The majority of patients in our forensic hospital setting enter from the jail setting for psychiatric reasons, having been found either incompetent to stand trial (IST) on an alleged criminal charge or having been adjudicated not guilty by reason of insanity (NGRI) for a criminal offence. These patients are admitted on court orders for psychiatric evaluation and treatment. In contrast, prison inmates are persons who have been convicted of a criminal offence and incarcerated for purposes such as containment for a determinate sentence of time, not for psychiatric treatment. As such, forensic psychiatric inpatients are qualitatively different from prison inmates based on the relevant legal findings and mental health issues present, and they are not admitted to this hospital solely on the diagnosis of ASPD alone; in fact, ASPD alone would militate against admission here. However, they share characteristics with prison inmates because they enter the forensic inpatient setting after being charged with a criminal offence, similar to how an inmate enters prison. In addition, some forensic inpatients may have previously been in the prison system on previous charges.

It was the impression of the unit staff on our male admission unit that those patients who have tattoos would frequently carry a diagnosis of ASPD or another Axis II diagnosis in addition to a diagnosis of a major Axis I psychiatric illness. As stated above, ASPD alone would not qualify for a judgment of NGRI or IST, so the diagnosis of ASPD is always comorbid with some other serious and significant Axis I psychopathology. The most common Axis I disorders diagnosed in these patients are psychotic disorders such as schizophrenia, schizoaffective disorder, delusional disorder, or major mood disorders such as bipolar or major depression with or without psychotic features. Not surprising for this setting, many patients also have diagnoses of substance abuse or dependence. Present less often are diagnoses of organic impairment or mental retardation.

This paper explores the hypothesis that forensic psychiatric patients who possess tattoos are significantly more likely to carry the diagnosis of ASPD than patients who do not possess tattoos. This hypothesis is generated by the question of what the meaning of the tattoo(s) might be for this particular subset of the forensic population. The focus of this study was on the presence and physical characteristics of tattoos. The premise is that the presence of a tattoo may help alert clinicians to the possible diagnosis of ASPD in these inpatients and may aid the clinician in treatment of this difficult patient population. Other differences between tattooed and non-tattooed patients were also explored, as were differences between individuals with ASPD and individuals without ASPD.

\section{Method}

\section{Participants}

Participants were obtained from the population of the male admission unit of a maximum-security state forensic psychiatric facility. This unit included two main groups of patients: criminal defendants awaiting trial who were determined by a court of law to be IST because of a mental defect (about $50 \%$ ), and those who had been adjudicated NGRI of a criminal offence (about 50\%). A total of 66 male patients sequentially admitted to the unit over a 5-month period in November 1995 to April 1996 were approached and asked to participate in a study that would 'examine the importance tattoos and other body markings have to people'. We obtained signed informed consent from each participant, and separate signed informed consent 
from participants who agreed to allow photographs of their tattoos. The study received approval from the institution's review board. There were no consequences, positive or negative, for the patients who agreed or declined to participate in the study. Participation in the study did not impact decisions regarding forensic, legal or clinical issues of a participant. The diagnostic and study findings were not available to the treating psychiatrist or social work clinician. Thirty-six patients $(55 \%)$ agreed to participate in the study. Patients who refused to participate usually cited lack of interest or poor motivation in that some just did not wish to volunteer for or join in anything that was not required of them. The non-participant group of 30 patients included 13 Caucasian (43\%) and 16 African American (53\%) individuals, and one whose race was 'unknown' (3\%). Non-participants' ages ranged from 28 to 82 years old ( $X=46.9$, standard deviation $(\mathrm{SD})=12.9)$. Two patients were excluded: one with severe acute psychosis and one with organic brain syndrome.

The final sample was composed of 23 Caucasian (64\%) and 13 African American (36\%) men. Participants' ages ranged from 20 to 59 years old $(X=33.7, \mathrm{SD}=10.3)$. The majority of participants were single $(67 \%)$ at the time of study entry, $8 \%$ were married, $6 \%$ were separated, $17 \%$ were divorced and $2 \%$ were widowed. Most also reported a history of perpetrating violent crime (78\%). Participants' educational background ranged from some high school to a graduate degree, with most reporting a high school degree (36\%) or some college/advanced training experience (42\%). The majority reported holding an unskilled job (47\%), with others reporting a skilled position (17\%), administrative position $(6 \%)$ or no current work $(30 \%)$ at the time of the offence.

\section{Measures}

Participants completed a semi-structured interview, the Tattoo Questionnaire (see Appendix A), which was developed for this study with the purpose of determining whether a patient pos- sessed tattoos or other decorative body markings. Items on the Tattoo Questionnaire helped to identify tattoos and to characterize them further. Data were gathered on each tattoo according to the patient's description of its location; appearance; the reason, circumstances and manner in which it was obtained; and attitude toward the tattoo (i.e. regretful, proud, etc.). If a participant had a tattoo, he was asked to show it to the interviewer; no participant declined to do so. No physical exam was conducted to establish the presence of a tattoo because, at the time of admission and as part of the routine physical examination done at admission to the CFP, each patient was assessed for the presence of tattoos and other body markings or piercings as part of a general security screening assessment. Additional information gathered during the interview included the patient's age and the number of other people present when the tattoo was obtained, whether the patient was intoxicated at the time and if the tattoo was done professionally. The surface area of each tattoo was also calculated by a two-dimensional measurement with a flexible tape measure performed by one of the authors (W. C.). The subjects' height and weight were used to determine their total body surface area (BSA). Items used for this study included those related to the presence or absence of a tattoo, number of tattoos, total tattooed BSA of each tattooed subject, percentage with professionally done tattoos and percentage of tattoos that were on visible locations of the body (i.e. hands, head or neck).

The diagnosis of ASPD was initially assessed through two different methods to reflect commonly employed methods of clinical diagnosis: a DSM-IV checklist for ASPD and the DSM-IV diagnosis at admission to the hospital as determined by the official DSM-IV diagnosis on the admission summary (APA, 1994). Admission diagnoses were standard clinical admission assessments made using DSM-IV criteria. Attending psychiatrists on the unit made the DSM diagnoses. The DSM-IV ASPD checklist was a checklist inventory that included all the DSM-IV criteria for ASPD, 
as well as the DSM-IV criteria for borderline personality disorder (BPD), and was completed by each patient's master's level social worker who was blind to the purpose of the study. Since the unit, a forensic psychiatric male admission unit, routinely admitted patients with personality disorders in addition to severe disorders of mood and/or psychosis, Axis II diagnoses were almost always considered on admission. However, because different attending psychiatrists might have a greater or lesser propensity to diagnose someone on Axis II, we used results on the checklist alone to determine inclusion or exclusion into the ASPD grouping.

General background information was obtained from each subject via a Demographic Data Questionnaire. This included, but was not limited to, demographic characteristics, information on the history of substance abuse, sexual abuse, selfmutilation (apart from tattooing or scarification), sexually transmitted disease, suicide attempts, juvenile delinquency, violent crime and family members with tattoos.

\section{Results}

Fifteen participants (42\%) possessed tattoos. Seventeen (47\%) participants met the criteria for ASPD according to the DSM-IV ASPD checklist, and five of these participants also received an admission diagnosis of ASPD. A decision was made to include all 17 participants with a positive DSMIV ASPD checklist in the ASPD group for data analysis because it was more inclusive and was believed to be more reliable than the admission diagnoses. Using a p value of 0.05 to define statistical significance, chi-squared analyses revealed that significantly more inpatients with tattoos had a diagnosis of ASPD than patients without tattoos, $\chi^{2}(1)=7.03, p<0.01$. Additional analyses explored other potential differences between patients with tattoos compared to those without tattoos. Patients with tattoos tended to be younger $(M=30.13$ years $)$ than non-tattooed patients $(M=36.19), t(34)=$ 1.80, $p<0.10$, and had less skilled occupations, $t(34)=2.23, p<.05$. No group differences were found on race, marital status or education. Results revealed that patients with tattoos were significantly more likely to have histories of substance abuse, $\chi^{2}(1)=8.23, p<0.01$, sexual abuse, $\chi^{2}(1)$ $=5.14, p<0.05$, and suicide attempts, $\chi^{2}(1)=7.03$, $p<0.01$ compared to non-tattooed individuals. Tattooed inpatients were no more likely to be diagnosed with BPD or have histories of juvenile delinquency, violent crime, family members with tattoos, self-mutilation or sexually transmitted disease compared to patients without tattoos. See Table 1.

Table 1: Differences between patients with and without tattoos on study variables

\begin{tabular}{lccl}
\hline Variable & Tattoo $(n=15)$ & No tattoo $(n=21)$ & Statistic \\
\hline Presence of ASPD & 11 & 6 & $\chi^{2}=7.03 * *$ \\
History of & & & $\chi^{2}=1.92$ \\
Borderline PD & 6 & 4 & $\chi^{2}=8.23^{* *}$ \\
Substance abuse & 14 & 10 & $\chi^{2}=1.84$ \\
Delinquency & 5 & 3 & $\chi^{2}=0.95$ \\
Violent crime & 11 & 6 & $\chi^{2}=2.26$ \\
Tattoos in family & 8 & 3 & $\chi^{2}=1.84$ \\
Self-mutilation & 5 & 1 & $\chi^{2}=5.14^{*}$ \\
Sexual abuse & 5 & 3 & $\chi^{2}=1.84$ \\
STD & 5 & 6 & $\chi^{2}=7.03 * *$ \\
Suicide attempt & 11 & & \\
\hline
\end{tabular}

$* p<0.05 ; * * p<0.01$.

ASPD, antisocial personality disorder; PD, personality disorder; STD, sexually transmitted disease. 
Exploratory analyses were also conducted to examine possible differences on study variables between the ASPD and non-ASPD groups. Participants in the ASPD group did not significantly differ from those in the non-ASPD group on race, age, marital status, education or occupation. Independent $t$-tests revealed that patients with ASPD reported a significantly greater number of tattoos, $t(34)=2.11, p<0.05$, and a trend toward a greater percent of total BSA with tattoos, $t(34)=1.81, p<0.10$. Furthermore, tattooed individuals with ASPD tended to have more of their tattoos in visible locations compared to individuals without ASPD, $\chi^{2}(1)=5.42, p<0.10$. The ASPD and non-ASPD groups did not differ, however, on likelihood of having professional tattoos. In terms of background history, patients with ASPD were significantly more likely to report a substance abuse history, $\chi^{2}(1)=6.74, p<0.01$. The groups did not differ on self-reported history of delinquency, violent crime, family members with tattoos, self-mutilation, sexual abuse, sexually transmitted disease and suicide attempts. See Table 2.

\section{Discussion}

This study was designed to test the hypothesis that inpatients in a forensic psychiatric facility who possess tattoos are more likely to have a diagnosis of ASPD than patients who do not have tattoos. This hypothesis was supported by the data. In our sample, significantly more forensic psychiatry inpatients with tattoos had ASPD than inpatients without tattoos. Conversely, subjects with ASPD also had a significantly higher number of tattoos, as well as a trend toward having tattoos cover a greater percent of their body and having tattoos in more visible locations. However, there were no differences between the ASPD and non-ASPD groups on a number of demographic measures or on whether or not tattoos were done professionally. In addition, tattooed subjects, with or without the diagnosis of ASPD, were significantly more likely to have histories of substance abuse, sexual abuse and suicide attempts. These findings suggest that clinicians should be alert to inquiring into these areas when assessing inpatients in a forensic psychiatric setting who have tattoos. Perhaps, more

Table 2: Differences between patients with and without ASPD on study variables

\begin{tabular}{llll}
\hline Variable & ASPD $(n=17)$ & Not ASPD $(n=19)$ & Statistic \\
\hline Number of tattoos & $\chi=2.65$ & $\chi=0.26$ & $t=2.11^{* *}$ \\
\% of BSA tattooed & $X=0.58$ & $\chi=0.11$ & $t=1.81^{*}$ \\
\% having more than $50 \%$ tattoo visibility & $33 \%$ & $25 \%$ & $\chi^{2}=5.42^{*}$ \\
\% with professional tattoos & $55 \%$ & $25 \%$ & $\chi^{2}=1.03$ \\
History of & & & \\
Substance abuse & 15 & 9 & $\chi^{2}=6.74^{* * *}$ \\
Delinquency & 5 & 3 & $\chi^{2}=0.96$ \\
Violent crime & 12 & 6 & $\chi^{2}=1.22$ \\
Tattoos in family & 8 & 3 & $\chi^{2}=0.91$ \\
Self-mutilation & 5 & 2 & $\chi^{2}=0.96$ \\
Sexual abuse & 4 & 4 & $\chi^{2}=1.09$ \\
STD & 4 & 7 & $\chi^{2}=0.03$ \\
Suicide attempt & 10 & & $\chi^{2}=1.74$ \\
\hline
\end{tabular}

$* p<0.10 ; * *<<0.05 ; * * * p<0.01$.

ASPD, antisocial personality disorder; BSA, body surface area; STD, sexually transmitted disease. 
importantly, these results suggest that tattoos may be an important diagnostic indicator of possible ASPD.

These findings appear consistent with the few existing studies to date examining tattoos and personality disorder in psychiatric and prison populations (Buhrich \& Morris, 1982; Gittleson et al., 1969; Manuel \& Retzlaff, 2002). One possible explanation for our finding is that tattoos may serve as an 'exoskeletal defense' for forensic inpatients to defend against perceived danger in the world and to strengthen one's ego definition (Grumet, 1983; Popplestone, 1963). Another consideration is that tattoos may serve as external manifestations of internal psychological and personality constructs, which patients with ASPD then utilize to express, pictorially, certain emotions because they are typically unable to express them verbally, interpersonally or individually (Grumet, 1983).

Given that tattoos appear to be much more common in the general population today than they were 10 years ago, at the time that this study was undertaken, it is possible that the presence or absence of a tattoo may have different meanings to different populations today than when the study was performed. We did a brief survey in 2005 and found that $40 \%$ of the patients currently on the unit where the original study was conducted possessed tattoos, which is not meaningfully different from the rate of $42 \%$ found at the time of the study. Thus, while the prevalence of tattoos in the general population appears to have increased, the prevalence of tattoos in forensic psychiatric inpatients has not changed much over this time. It is likely that the meaning or motivation of tattoos may also differ depending on the nature of the group under investigation. From a sociological point of view, the rising increase of tattoos in the general population suggests multiple meanings and motivations associated with tattoos. For example, the observation has been made that many forensic security aides and other hospital staff working with forensic psychiatric inpatients may have tattoos themselves, reflecting the overall increase of tattoos in general.
Perhaps, although we do not possess these data, the tattoo of a staff person would be of higher quality and mean something different to the person than that of a forensic psychiatric inpatient, who often possesses a homemade or crudely made tattoo that was either self-administered or produced with improvised means by an associate. However, as Demello (1993) described, the fine lined black only (monochromatic) tattoo typically recognized as a prison or street tattoo is seen more commonly in mainstream tattooing. It is interesting to consider the possible motivations that someone who works with forensic psychiatric inpatients has for obtaining a tattoo of their own, as well as those of other groups of people such as general psychiatric patients or the general population. Because this study focused only on inpatients in a forensic psychiatric facility, conclusions about the presence and meaning of tattoos on individuals in other segments of the population cannot be made from these results.

Following from our data, the presence of a tattoo on a patient in a forensic psychiatric hospital suggests a need for further exploration as to whether or not the patient possesses antisocial personality features or an ASPD diagnosis. Certainly, attention to the possible ASPD diagnosis might inform assessment and treatment planning. It may, in turn, reveal something about the object relations of the patient. In our earlier study on transitional objects and BPD, we found that in a general psychiatric inpatient population, those patients who brought teddy bears or other so-called transitional objects with them to the hospital were significantly more likely to have BPD than patients who did not bring such objects (Cardasis, Hochman, \& Silk, 1997). In maximum-security settings, patients are generally not allowed to bring with them any personal belongings that could be considered transitional objects by nature. They are often observed, however, to possess tattoos that perhaps might serve in the role of transitional objects. Of course, more would need to be understood as to when and under what circumstances the tattoos were obtained. 
This study is limited by several factors. First, different diagnoses other than ASPD may be associated with possession of tattoos. Although we did not find differences on history of BPD, it may be that tattoos are related to some other type of personality pathology.

Second, two different methods to determine ASPD were employed initially: clinical assessment by a psychiatrist (admission diagnosis), as well as a checklist of symptoms performed blindly by individuals not involved in the study but who had gotten to know the patient over a longer period of time. The DSM-IV ASPD checklist was completed by the social worker clinician after some period of time on the unit during which patterns of behaviour and interpersonal interactions could add to the assessment. This provided more data than was available at admission when the DSM-IV diagnosis was made. While blind to the purpose of the study, we do not know whether the checklist rater was influenced by noticing that the patient did or did not have a tattoo. The reader may also note the much higher rate of ASPD according to the DSMIV checklist (17 participants) compared to the admission diagnosis of ASPD (5 participants). The average length of stay of a patient in the CFP was several months to several years depending on whether the patient was admitted as IST or NGRI. Patients were approached about participating in the study several days or weeks after admission to the hospital. Thus, we believe that the discrepancy in diagnoses between the DSM-IV checklist and admission diagnosis could be the result of the social worker clinician having more data and background information about the patient when completing the DSM-IV ASPD checklist than the attending psychiatrist making a diagnostic assessment on the day of admission. We used the checklist diagnosis of ASPD because we felt it included more data in its diagnostic determination.

Third, the study is limited by the relatively small sample size which makes the determination of statistical significance between the reference groups more difficult. It may be that more differences between groups would be found with a larger sample. Finally, because eligible African American males appeared to be less likely to consent to being part of this study (out of the total number approached), it is possible that our findings do not adequately represent African American men's experiences in this particular forensic setting. Similarly, our sample did not include female forensic inpatients.

Despite these issues, it seems that male forensic psychiatric inpatients with tattoos should be carefully assessed for the presence of ASPD as well as for substance abuse, sexual abuse and suicide attempts. Future directions for research in this area might include whether adolescents with tattoos are more likely to have conduct disorder than adolescents without tattoos, as well as determining the age at which the tattoo was obtained, the motivation for obtaining the tattoo and the life events that were occurring at the time the tattoo was received. A further inquiry into the meaning and subject content of the tattoo to its bearer and additional information about the attitude the individual has toward the tattoo, and whether he wishes to have one or more tattoos modified, covered with another tattoo or removed, would also be of interest.

Overall, we think that this study may provide additional clues to the diagnosis of ASPD among a forensic population. We further believe that knowledge of ASPD as a possible diagnosis, alongside other psychiatric diagnoses, may be informative for the treatment planning, the treatment approach and actual treatment of this population.

\section{References}

American Psychiatric Association (APA). (1980). Diagnostic and statistical manual of mental disorders (3rd ed.). Washington, DC: American Psychiatric Association.

American Psychiatric Association (APA). (1994). Diagnostic and statistical manual of mental disorders (4th ed.). Washington, DC: American Psychiatric Association.

Anderson, R. R. (2006). Commentary: Tattoos and body piercings. Journal of the American Academy of Dermatology, 55,422 . 
Braithwaite, R., Robillard, A., Woodring, T., Stephens, T., \& Arriola, K. J. (2001). Tattooing and body piercing among adolescent detainees: Relationship to alcohol and other drug use. Journal of Substance Abuse, 13, 5-16.

Buhrich, N., \& Morris, G. (1982). Significance of tattoos in male psychiatric patients. Australian and New Zealand Journal of Psychiatry, 16, 185-189.

Cardasis, W., Hochman, J. A., \& Silk, K. R. (1997). Transitional objects and borderline personality disorder. American Journal of Psychiatry, 154, 250-255.

Cleckley, H. (1950). The mask of sanity. St. Louis: CV Mosby.

Demello, M. (1993). The convict body: Tattooing among male American prisoners. Anthropology Today, 9, 10-13.

Fisher, S. (1963). A further appraisal of the body boundary concept. Journal of Consulting Psychology, 27, 62-74.

Gittleson, N. L., Wallen, G. D. P., \& Dawson-Butterworth, K. D. (1969). The tattooed psychiatric patient. British Journal of Psychiatry, 115, 1249-1253.

Grumet, G. W. (1983). Psychodynamic implications of tattoos. American Journal of Orthopsychiatry, 53, 482-492.

Hare, R. D. (2006). Psychopathy: A clinical and forensic overview. Psychiatric Clinics of North America, 29, 709-724.

Hare, R. D., \& Neumann, C. S. (2005). Structural models of psychopathy. Current Psychiatry Reports, 7, 57-64.

Hare, R. D., Hart, S. D., \& Harpur, T. J. (1991). Psychopathy and the DSM-IV criteria for antisocial personality disorder. Journal of Abnormal Psychology, 100, 391-398.

Hill, C. D., Neumann, C. S., \& Rogers, R. (2004). Confirmatory factor analysis of the psychopathy checklist: Screening version in offenders with axis I disorders. Psychological Assessment, 16, 90-95.

Kernberg, O. F. (1976). Object relations theory and clinical psychoanalysis. New York: Jason Aronson.

Kokkevi, A., Stefanis, N., Anastasopoulou, E., \& Kostogianni, C. (1998). Personality disorders in drug abusers:
Prevalence and their association with AXIS I disorders as predictors of treatment retention. Addictive Behaviors, 23, 841-853.

Laumann, A. E., \& Derrick, A. J. (2006). Tattoos and body piercings in the United States: A national data set. Journal of the American Academy of Dermatology, 55, 413-421.

MacKinnon, R. A., \& Michels, R. (1971). The psychiatric interview in clinical practice. Philadelphia, PA: WB Saunders Company.

Manuel, L., \& Retzlaff, P. D. (2002). Psychopathology and tattooing among prisoners. International Journal of Offender Therapy $\mathcal{E}$ Comparative Criminology, 46/5, 522-531.

Measey, L. G. (1972). The psychiatric and social relevance of tattoos in royal navy detainees. British Journal of Criminology, 19, 181-186.

Popplestone, J. (1963). A syllabus of the exoskeletal defenses. Psychological Record, 13, 15-25.

Reich, J. (1997). Antisocial traits in psychiatrically ill veterans without antisocial personality disorder: Relationship to Axis I disorders and effects on functioning. Psychiatry Research, 71, 77-82.

Ross, S., Dermatis, H., Levounis, P., \& Galanter, M. (2003). A comparison between dually diagnosed inpatients with and without Axis II comorbidity and the relationship to treatment outcome. American Journal of Drug $\mathcal{E}$ Alcohol Abuse, 29, 263-279.

Wohlrab, S., Stahl, J., \& Kappeler, P. M. (2007). Modifying the body: motivation for getting tattooed and pierced. Body Image, 4, 87-95.

Address correspondence to: William Cardasis, MD, Admissions Unit Director, Center for Forensic Psychiatry, Michigan Department of Community Health, PO Box 2060, Ann Arbor, Michigan, 48106, USA. Email: kardasis@umich.edu 


\section{Appendix A}

\section{TATTOO QUESTIONNAIRE}

\begin{tabular}{|c|c|c|}
\hline \multicolumn{3}{|l|}{ Name: } \\
\hline \multicolumn{3}{|l|}{ Height: } \\
\hline \multicolumn{3}{|l|}{ Weight: } \\
\hline Do/did you have a tattoo(s) or any other decorative body markings (i.e. branding, piercing?) & Yes & No \\
\hline \multicolumn{3}{|l|}{ How many times have you been tattooed, branded or pierced? } \\
\hline \multicolumn{3}{|l|}{ How many tattoos currently present? } \\
\hline \multicolumn{3}{|l|}{ Why did you get tattooed—motivation, reason? } \\
\hline Do any of the tattoos have special meaning to you (i.e. magical, ward off evil, religious, etc.)? & Yes & No \\
\hline \multicolumn{3}{|l|}{ Describe, including number of tattoo: } \\
\hline Do any of the tattoos have sexual significance to you? & Yes & No \\
\hline \multicolumn{3}{|l|}{ Describe, including number of tattoo: } \\
\hline \multicolumn{3}{|l|}{ What is your attitude toward the tattoo(s) (i.e. regretful, proud, etc.)? } \\
\hline \multicolumn{3}{|l|}{ Describe, including number of tattoo: } \\
\hline Have you ever had a tattoo removed? & Yes & No \\
\hline \multicolumn{3}{|l|}{ Describe: } \\
\hline When was your first arrest in relation to the first tattooing? & Before & After \\
\hline Does urticaria occur in any tattoo when upset, angry, etc.? & Yes & No \\
\hline Self-rating of tattoos in order of significance: & & \\
\hline
\end{tabular}

\begin{tabular}{|c|c|c|c|c|c|c|c|c|c|c|}
\hline \multicolumn{8}{|c|}{ Please describe (first/most special) tattoo in more detail. } & \multicolumn{3}{|l|}{ Age obtained: } \\
\hline Location: & forearm & & $\begin{array}{l}\text { upper } \\
\text { arm }\end{array}$ & chest & $\begin{array}{l}\text { leg/ } \\
\text { foot }\end{array}$ & back & $\begin{array}{l}\text { head/ } \\
\text { neck }\end{array}$ & genitals & hand & mucous \\
\hline \multicolumn{4}{|c|}{ dimensions $(\mathrm{cm})$ : } & \multicolumn{3}{|c|}{ surface area $\left(\mathrm{cm}^{2}\right)$ : } & \multicolumn{2}{|c|}{ including $>1$ body part? } & yes & no \\
\hline \multicolumn{6}{|l|}{ design: } & \multicolumn{3}{|c|}{ visible with clothes? } & yes & no \\
\hline \multicolumn{6}{|l|}{ color(s): } & \multicolumn{3}{|c|}{ done professionally/paid for it? } & yes & no \\
\hline intoxicated? & yes & no & Etoh & MJ & $\mathrm{Coc}$ & Her & Stim & Dep & Hall & Oth: \\
\hline \multicolumn{6}{|c|}{ Number of people present (besides tattooist): } & \multicolumn{5}{|c|}{ life circumstances (jail, military, etc.): } \\
\hline
\end{tabular}




\begin{tabular}{|c|c|c|c|c|c|c|c|c|c|c|}
\hline \multicolumn{8}{|c|}{ Please describe $\# 2$ tattoo in more detail. } & \multicolumn{3}{|c|}{ Age obtained: } \\
\hline Location: & forearm & & $\begin{array}{l}\text { upper } \\
\text { arm }\end{array}$ & chest & $\begin{array}{l}\text { leg/ } \\
\text { foot }\end{array}$ & back & $\begin{array}{l}\text { head/ } \\
\text { neck }\end{array}$ & genitals & hand & mucous \\
\hline \multicolumn{4}{|c|}{ dimensions $(\mathrm{cm})$ : } & \multicolumn{3}{|c|}{ surface area $\left(\mathrm{cm}^{2}\right)$ : } & \multicolumn{2}{|c|}{$\begin{array}{l}\text { including }>1 \text { body } \\
\text { part? }\end{array}$} & yes & no \\
\hline \multicolumn{6}{|l|}{ design: } & \multicolumn{3}{|c|}{ visible with clothes? } & yes & no \\
\hline \multicolumn{6}{|l|}{ color(s): } & \multicolumn{3}{|c|}{$\begin{array}{l}\text { done professionally/paid for } \\
\text { it? }\end{array}$} & yes & no \\
\hline intoxicated? & yes & no & Etoh & MJ & Coc & Her & Stim & Dep & Hall & Oth: \\
\hline \multicolumn{6}{|c|}{ Number of people present (besides tattooist): } & \multicolumn{5}{|c|}{ life circumstances (jail, military, etc.) } \\
\hline
\end{tabular}

\begin{tabular}{|c|c|c|c|c|c|c|c|c|c|c|}
\hline \multicolumn{8}{|c|}{ Please describe $\# 3$ tattoo in more detail. } & \multicolumn{3}{|c|}{ Age obtained: } \\
\hline Location: & forearm & & $\begin{array}{l}\text { upper } \\
\text { arm }\end{array}$ & chest & $\begin{array}{l}\text { leg/ } \\
\text { foot }\end{array}$ & back & $\begin{array}{l}\text { head/ } \\
\text { neck }\end{array}$ & genitals & hand & mucous \\
\hline \multicolumn{4}{|c|}{ dimensions $(\mathrm{cm})$ : } & \multicolumn{3}{|c|}{ surface area $\left(\mathrm{cm}^{2}\right)$ : } & \multicolumn{2}{|c|}{$\begin{array}{l}\text { including }>1 \text { body } \\
\text { part? }\end{array}$} & yes & no \\
\hline \multicolumn{6}{|l|}{ design: } & \multicolumn{3}{|c|}{ visible with clothes? } & yes & no \\
\hline \multicolumn{6}{|l|}{ color(s): } & \multicolumn{3}{|c|}{ done professionally/paid for it? } & yes & no \\
\hline intoxicated? & yes & no & Etoh & MJ & Coc & Her & Stim & Dep & Hall & Oth: \\
\hline \multicolumn{6}{|c|}{ Number people present (besides tattooist): } & \multicolumn{5}{|c|}{ life circumstances (jail, military, etc.): } \\
\hline
\end{tabular}

